\title{
Evaluation of record linkage of two large administrative databases in a middle income country: stillbirths and notifications of dengue during pregnancy in Brazil
}

Enny S Paixão ${ }^{1 *}$ (B), Katie Harron ${ }^{1}$, Kleydson Andrade ${ }^{2}$, Maria Glória Teixeira ${ }^{2}$, Rosemeire L. Fiaccone ${ }^{3}$, Maria da Conceição N. Costa $^{2}$ and Laura C. Rodrigues ${ }^{1}$

\begin{abstract}
Background: Due to the increasing availability of individual-level information across different electronic datasets, record linkage has become an efficient and important research tool. High quality linkage is essential for producing robust results. The objective of this study was to describe the process of preparing and linking national Brazilian datasets, and to compare the accuracy of different linkage methods for assessing the risk of stillbirth due to dengue in pregnancy.

Methods: We linked mothers and stillbirths in two routinely collected datasets from Brazil for 2009-2010: for dengue in pregnancy, notifications of infectious diseases (SINAN); for stillbirths, mortality (SIM). Since there was no unique identifier, we used probabilistic linkage based on maternal name, age and municipality. We compared two probabilistic approaches, each with two thresholds: 1) a bespoke linkage algorithm; 2) a standard linkage software widely used in Brazil (ReclinkIII), and used manual review to identify further links. Sensitivity and positive predictive value (PPV) were estimated using a subset of gold-standard data created through manual review. We examined the characteristics of false-matches and missed-matches to identify any sources of bias.

Results: From records of 678,999 dengue cases and 62,373 stillbirths, the gold-standard linkage identified 191 cases. The bespoke linkage algorithm with a conservative threshold produced 131 links, with sensitivity $=64.4 \%$ (68 missed-matches) and PPV $=92.5 \%$ (8 false-matches). Manual review of uncertain links identified an additional 37 links, increasing sensitivity to $83.7 \%$. The bespoke algorithm with a relaxed threshold identified 132 true matches (sensitivity $=69.1 \%$ ), but introduced 61 false-matches (PPV $=68.4 \%$ ). Reclinklll produced lower sensitivity and PPV than the bespoke linkage algorithm. Linkage error was not associated with any recorded study variables.

Conclusion: Despite a lack of unique identifiers for linking mothers and stillbirths, we demonstrate a high standard of linkage of large routine databases from a middle income country. Probabilistic linkage and manual review were essential for accurately identifying cases for a case-control study, but this approach may not be feasible for larger databases or for linkage of more common outcomes.
\end{abstract}

Keywords: Data linkage, Routine data, Electronic health records, Linkage quality, Linkage accuracy, Stillbirth, Dengue

\footnotetext{
* Correspondence: enny.cruz@lshtm.ac.uk

${ }^{1}$ London School of Hygiene and Tropical Medicine, Keppel St, Bloomsbury,

London WC1E 7HT, UK

Full list of author information is available at the end of the article
} 


\section{Background}

Record linkage is the process used to bring together information recorded in different sources about the same individual or group of individuals [1]. Due to the growing availability of administrative population-based health databases, linkage has become an efficient and important research tool [2]. One research area for which linkage is particularly important is maternal and infant health, where linkage can facilitate increased understanding of how maternal health trajectories prior to and during pregnancy are associated with birth and later childhood outcomes [3]. Many countries use linkage of records from mothers and their babies to underpin both research and service evaluation [4-6].

High quality linkage is required so that robust results can be obtained from linked data. However, there are specific challenges in mother-baby linkage, particularly concerning data from low-middle income countries. Firstly, quality of linkage is limited by the availability of unique or well-completed identifiers, and by data quality (including truncated records and absent or ambiguous information [7, 8]. A further complication is that linkage of records belonging to different individuals is required (e.g. mothers and their babies), which limits the availability of common identifiers for linkage. Finally, when datasets do not overlap exactly, the expected number of matches is unknown, making it difficult to establish the expected number of records that should be linked [3].

There are two main approaches to linkage: deterministic and probabilistic. Deterministic linkage is a rulebased approach, typically using a unique identifier or set of common identifiers present in both files. Probabilistic linkage is useful when the quality of identifiers is not sufficient for a strict deterministic linkage due to missing values or typographical errors [9]. Probabilistic linkage combines evidence across a number of identifiers such as name, age and place of residence to calculate a match weight, representing the likelihood that two records belong to the same person, i.e., that they are a "true match" [10]. Match weights are used to classify records as links, non-links and uncertain links, generally by defining two thresholds [11]. The choice of threshold affects the number of "false-matches" (records from different individuals that are linked) and "missed-matches" (records from the same individual that fail to link) [12]. Probabilistic linkage has facilitated many studies in countries without a unique identifier [4-6].

The impact of linkage errors, in terms of bias in results of analysis, depends on the study in question [13]. For example, in many studies, it may be important to achieve a high match rate, so that the resulting linked data is representative of the source population and to avoid selection bias (especially if missed-matches are non-random, i.e. are more likely to occur in specific subgroups of records). In others, it may be more important to avoid false-matches. For example, in case-control studies, we may be more concerned with accurately establishing exposure status through linkage with a disease registry. This would require certainty that linked records really should have been linked, and unlinked records did not have the exposure.

In this study, we aimed to use linkage of national data sources to facilitate a case-control study of stillbirths in women who had notified symptomatic dengue during pregnancy. Linkage was required to establish exposure status in cases. In this scenario, it was important to: 1) prioritize true matches (i.e. high specificity of the exposure), whilst high sensitivity was less important; 2) retain sufficient cases for a reasonable sample size; and 3) verify absence of bias (i.e. understand whether any groups were more or less likely to be linked). This study presents an approach to preparing and linking mortality and morbidity data from routine data sources in Brazil, comparing the performance of different linkage methods for identifying exposure of pregnant women to dengue.

\section{Methods \\ Datasets}

We linked two routinely collected data sets: for dengue, notifications of infectious diseases (SINAN); for stillbirths, mortality (SIM), for 2009 and 2010.

1. SINAN: Notifiable Diseases Information System (Sistema de Informação de Agravos de Notificação/ SINAN), containing individual-level data on all notified diseases.

\section{Source}

The Brazilian Ministry of Health has required notification of all cases of dengue seen in health facilities in Brazil. SINAN captures clinical cases of disease, through forms completed by any health professional who suspects dengue; this notification is compulsory in the country. After a dengue suspected case is identified, the Epidemiological Surveillance Service investigates cases in order to confirm or discard the suspicion based on laboratory results and the Brazilian definition of clinical epidemiological criteria of dengue: presence of fever and two or more of the following symptoms (retro-orbital or ocular pain, headache, rash, myalgia, arthralgia, leukopenia, or haemorrhagic manifestations) [14]. Forms include personal information on the patient (name, place of residence, age, marital status, and education) and on their disease (symptoms, laboratory tests and severity). 


\section{Completeness of linkage variables}

Maternal name was $99.5 \%$ complete; municipality was $100 \%$ complete. Where age in years was missing $(1.3 \%$ of records), we derived age from date of birth.

\section{Data extraction}

Data were extracted from SINAN for all suspected dengue cases ( $n=1,981,912$ individuals). We excluded records for 900,054 men, and 398,710 cases discarded by the Epidemiological Surveillance services (Fig. 1).

2. SIM: Mortality Information System (Sistema de Informação sobre Mortalidade), containing individual-level data on all deaths including stillbirths.

\section{Source}

The Brazilian Ministry of Health requires notification of all patients who die, irrespective of place of death. Deaths are recorded using a death certificate, which is a legal document completed by physicians [15]. The definition of stillbirth as recorded in SIM is the death of a product of conception before the expulsion or complete extraction from the body of the pregnant woman, occurring from 22 weeks or weighing more than $500 \mathrm{~g}$ [15].
The form includes information on the mother (name, place of residence, age, marital status, education, whether she had a previous stillbirth or a child who died); and the pregnancy (length of gestation, type of delivery).

\section{Completeness of linkage variables}

Maternal name was complete in $98.8 \%$ of records; municipality was $100 \%$ complete. Age was complete in $84.6 \%$ of records.

\section{Data extraction}

Data on all deaths were extracted from SIM ( $n=2,303,893$ records). We kept only the stillbirths $(n=63,108)$.

\section{The process of linking}

Data pre-processing

We excluded records without names, those with generic names such as "unknown" or "stillbirth", records with only one name (e.g. "Maria"), and those name recorded as numbers: our final study population comprised 678,999 dengue notifications from SINAN and 62,373 stillbirths from SIM (Fig. 1). We searched automatically for improbable ages (e.g. 99 or age of the mother 1 year

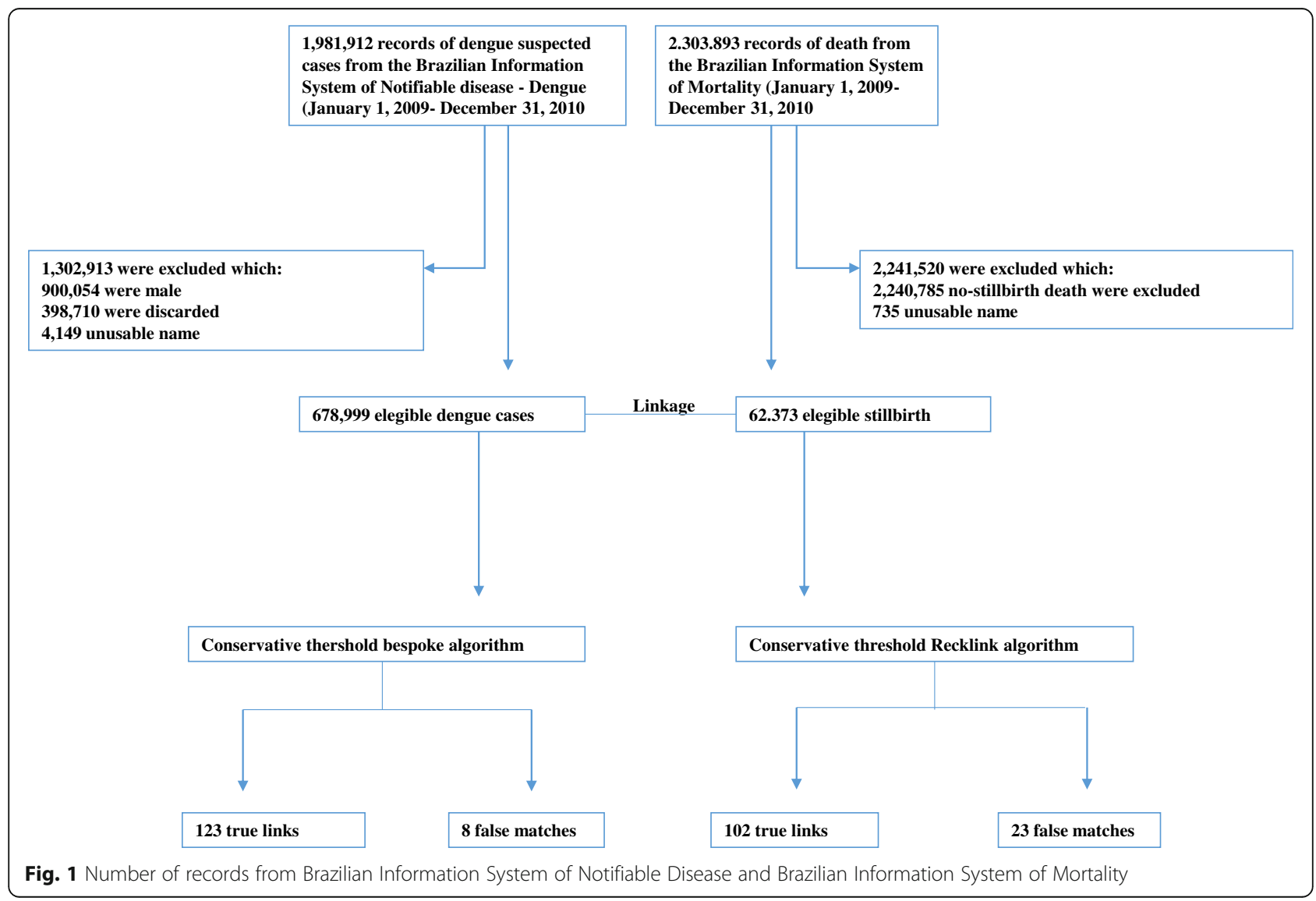


old) and set to null. We removed punctuation, deleted consecutive spaces, transformed known prefixes in the real names (e.g. Mr. $\rightarrow$ Maria), deleted unknown prefixes, replaced names in upper case and dropped middle initials.

In Brazil, full names usually have several components, and we aimed to retain the discriminatory power of this variable. In our data, the mean number of names per mother was three (maximum eight). We derived distinct variables for first name, second name, and last name. Similarity between names recorded in SIM and SINAN was compared using the Jaro-Winkler string comparator [16]. The Jaro-Winkler string comparator counts the number of common characters between two strings and the number of transpositions of these common characters, producing similarity values varying between 1 (perfectly similar) and 0 [16]. We categorized the string comparator score as $(0,0.85], \quad(0.85,0.9], \quad(0.9,0.95]$, $(0.95,0.99]$ and $(0.99,1]$.

\section{Blocking}

The total number of pairwise comparisons between SINAN and SIM would be prohibitively high: $184,922 \times 31,867=5,892,909,374$ in 2009 and $494,077 \times 30,506=15,072,312,962$ in 2010 (a dengue epidemic year). To decrease the number of pairwise comparisons, we only attempted to link women that resided in the same municipality $(n=5565$ municipalities in Brazil in 2010). This process is called blocking; blocking by municipality assumes that records from different municipalities do not belong to the same women. We used this blocking scheme because we considered municipality to be very reliable, and unlikely to have changed between dengue in pregnancy and end of pregnancy.

\section{Gold-standard data}

We created a "Gold-standard" dataset using nine steps (Table 1). Firstly, we linked SIM and SINAN using deterministic linkage with exact agreement on full name and age. Subsequent steps relaxed the rules. Since mothers could be diagnosed during pregnancy in 1 year and give birth in the following year, and to allow for errors in recording, we allowed matches where there were differences of up to 2 years in age. To account for differences in the ways names were recorded, we separately looked for agreement on first and last name, first and second name, or second and last name. Each step was followed by manual review to exclude false-matches. Since some names were very common in the database (e.g. Maria), records with these names were only considered as a match where there was exact agreement on name and municipality, and age differed by no more than 1 year.
Table 1 Deterministic rules used to create the gold-standard database

\begin{tabular}{ll}
\hline Linkage rule & Number of links (\%) \\
\hline Full name and age $^{\mathrm{a}}$ & $46(24.1 \%)$ \\
Age $^{\mathrm{a}}$ and combination of first and last name & $13(6.8 \%)$ \\
Age $^{\mathrm{a}}$ and combination of first and second name & $9(4.8 \%)$ \\
Age $^{\mathrm{a}}$ and combination of second and last name & $19(9.9 \%)$ \\
Full name & $65(34.0 \%)$ \\
First and last name & $19(9.9 \%)$ \\
First and second name & $8(4.2 \%)$ \\
Second and last name & $12(6.3 \%)$ \\
Age $^{\mathrm{a}}$ and Jaro-Winkler string comparator $>0.95$ & $0(0 \%)$ \\
& $\mathbf{1 9 1}$ \\
\hline
\end{tabular}

${ }^{a}$ Age in years as recorded in the data or as derived from date of birth

\section{Bespoke algorithm}

For each record pair we calculated a probabilistic match weight based on two conditional probabilities: the probability of agreement given records belong to the same mother-baby pair (m-probability; P (agreement|match)) and the probability of agreement given records belong to different mother-baby pairs (u-probability, P (agreement|non-match)).

M-probabilities for each identifier were estimated from the true matches in the gold-standard dataset. U-probabilities were calculated based on a list of nonmatches, created from all pairwise comparisons of records within SINAN, excluding those belonging to the same individual. We used the SIINAN database to calculate the u-probabilities, as it was the larger of the two databases.

Frequency-based weights were calculated for each category of Jaro-Winkler score comparator [16] (for name of the mother) and year of age. Weights were also separately calculated for the five most frequently occurring names in the data (Maria, Ana, Santos, Souza and Oliveira).

Since we were linking different subjects (the mother and her stillbirth), and because the exposure (dengue during pregnancy) could have happened up to 9 months before the outcome (stillbirth) there were timing issues to consider. Two records could differ in time by 9 months and could bridge over the calendar year; some mothers would have the birthday between the data of dengue and the date of the stillbirth. To allow for this, we estimated different weights according to the similarity of age across datasets: equal ages, age differing by 1 year, age differing by 2 years, and ages differing by more than 2 years (Table 2 ).

Match weights were calculated by summing the ratio of $\mathrm{m}$-probabilities and $\mathrm{u}$-probabilities across different identifiers [1]. The algorithm was implemented in Stata and R. 
Table 2 Comparison of linkage strategies for the bespoke algorithm and ReclinklII

\begin{tabular}{|c|c|c|}
\hline & Bespoke algorithm & ReclinkIII \\
\hline Manipulation of names & $\begin{array}{l}\text { - Multiple variables created for first name, second name, } \\
\text { and last name }\end{array}$ & - Variables created for first and last name \\
\hline Blocking & - Municipality & - Soundex for name + municipality \\
\hline Calculation of $m$ and $u$ probabilities & $\begin{array}{l}\text { - m-probability: calculated using true-matches in gold-standard } \\
\text { - u- probability: calculated using non-matches in SINAN }\end{array}$ & $\begin{array}{l}\cdot m \text {-probabilities }=0.9 \\
\cdot \boldsymbol{u} \text {-probabilities }=0.1\end{array}$ \\
\hline Match weight calculation & $\begin{array}{l}\text { - Separate weights calculated for the five most common names } \\
\text { - Agreement on name classified using Jaro-Winkler string comparator } \\
\text { - Different weights calculated according to closeness of age. }\end{array}$ & $\begin{array}{l}\text { - Did not account for common names } \\
\text { - Levenshtein string comparator } \\
\text { - Did not account for timing issues }\end{array}$ \\
\hline
\end{tabular}

\section{ReclinkIII algorithm}

As we were using Brazilian data, we compared the accuracy of our bespoke linkage algorithm with a widely used software for linking data in Brazil, called Reclink version III.

ReclinkIII calculates match weights in 3 steps: 1) Manipulation of names; 2) Blocking (Reclink matches pairs within blocks of similar names defined by soundex; in addition we programmed ReclinkIII to block by municipality); 3) Match weight calculation (the final score is the sum of the weighted scores of each field, e.g. name and age).

ReclinkIII applies the Levenshtein string comparator to compare names [17]. The Levenshtein string comparator is defined as the minimum number of insertions, deletions, or substitutions necessary to change one string into the other, the values varying between 1 (perfect similarly) and 0 (total disagreement). The $\mathrm{m}$-probabilities and $\mathrm{u}$-probabilities were based on default values as suggested by the software: m-probabilities $=0.9$ and $\mathrm{u}$ probabilities $=0.1$ for all identifiers (Table 2) [18]

\section{Classification of links}

Records pairs were ordered by match weight and manually inspected to identify threshold values to classify comparison pairs as non-links, links and uncertain links. The number of expected matches was unknown, because we did not know a priori how many of the mothers in SIM should link to a stillbirth in SINAN. Therefore, we explored two different threshold choices for each algorithm. We first chose a conservative threshold, aiming to exclude as many as false-matches as possible (high positive predictive value). We then chose a relaxed threshold, aiming to capture as many of the true matches as possible (high sensitivity). Any records above the cut-off threshold were classified as links. For the best performing approach, we manually inspected uncertain links to determine whether or not they belonged to the same mother-baby pair.

\section{Statistical analysis}

For both the bespoke and the ReclinkIII algorithm, and for each threshold (conservative and relaxed), we estimated the sensitivity and positive predictive value (PPV) by comparing linkage results with the goldstandard dataset. Since we expected the number of links to be very small in comparison to the size of the datasets, we did not calculate specificity or negative predictive value (as these measures would be consistently high). To account for in-sample optimism, we also present average estimates based on 'leave one out' cross classification.

For the best performing algorithm, we examined which characteristics were associated with false-matches and missed matches. Categorical variables were compared between groups with $\mathrm{Chi}^{2}$ test or Fisher's exact test. A two-sided $P$ value of less than 0.05 was considered to indicate statistical significance. We examined maternal age $(<20,20-35,>35$ years), maternal education (illiterate, 1-3 years, 4-7 years, 8-11 years and more than 11 years), previous stillbirths or abortions (yes/no), gestational age (less than 22 weeks, 22-27 weeks, 2831 weeks, 32-36 weeks, 37-41 weeks, more than 42 weeks) and weight when the stillbirth occurred ( $>=2500,1500-2500,<1500$ g). Stata version 14.1 was used for the statistical analyses.

\section{Results}

\section{Gold-standard}

Of the 678,999 eligible dengue cases in SINAN for 2009-2010, 191 were linked to a stillbirth record using the nine-step gold-standard algorithm (Table 1).

\section{Bespoke algorithm}

The conservative threshold was set to a match weight of 21, and resulted in 131 links (Fig. 1). Comparison with the gold-standard identified 8 false matches and 68 missed-matches, giving a sensitivity of $123 / 191=64.4 \%$ and a PPV of $123 / 131=93.9 \%$ (Table 3). Adjusting for in-sample optimism gave values of $64.4 \%$ and $94.1 \%$ respectively.

The relaxed threshold was set at 20 and resulted in 193 links. Comparison with the gold-standard identified 132 true links, giving a sensitivity of $132 / 191=69.1 \%$, and 61 false-matches, giving a PPV of $132 / 193=68.4 \%$ 
Table 3 Performance of linkage algorithms and thresholds

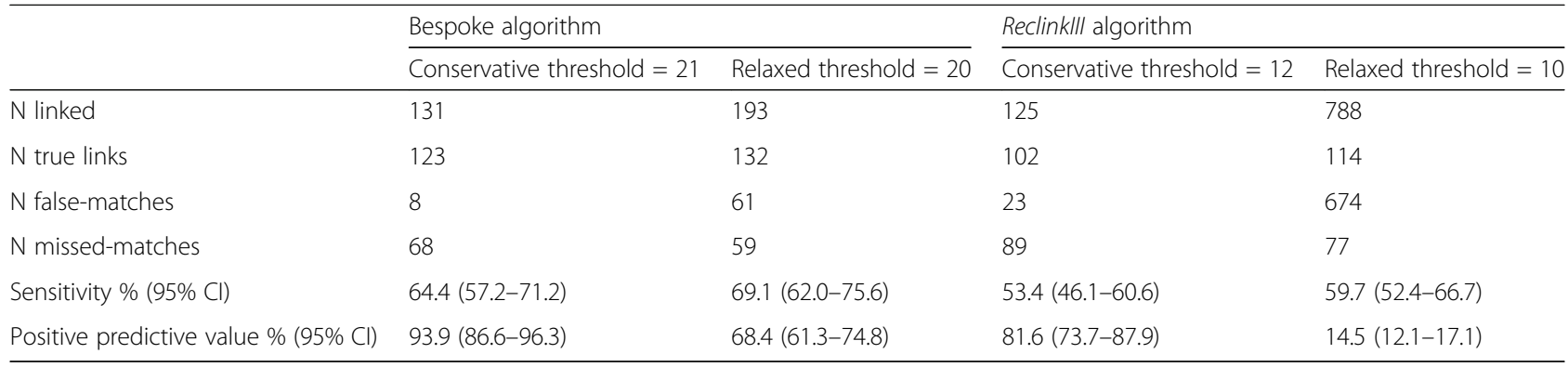

(Table 3). Adjusting for in-sample optimism gave values of $69.1 \%$ and $68.4 \%$ respectively.

\section{ReclinkIII}

The conservative threshold was set at 12 and resulted in 125 links (Fig. 1). Comparison with the gold-standard identified 102 true links, giving a sensitivity of 102/ $191=53.4 \%$, and 23 false-matches, giving a PPV of 102/ $125=81.6 \%$ (Table 3). Adjusting for in-sample optimism gave values of $53.4 \%$ and $81.8 \%$ respectively.

The relaxed threshold was set at 10 and resulted in 788 links. Comparison with the gold-standard identified 114 true links, giving a sensitivity of $114 / 191=59.7 \%$, and 674 false-matches, giving a PPV of $114 / 788=14.5 \%$ (Table 3). Adjusting for in-sample optimism gave values of $59.7 \%$ and $14.4 \%$ respectively.

\section{Linkage errors}

Missed-matches and false-matches had a higher proportion of missing data (Table 4). Linkage errors were not associated with any recorded study variables, although there was a suggestion that mothers aged $<20$ were slightly less likely to link ( $p=0.047)$ (Table 4$)$.

\section{Manual review}

For the bespoke linkage algorithm with the conservative threshold, uncertain links were defined as those with weights between 16 and 21. Records with these weights were classified through manually inspecting each record to determine whether or not they belonged to the same person. This added 37 links, resulting in 160 true links, increasing the sensitivity to $83.7 \%$ and bringing the total number of linked records to 168 .

\section{Discussion}

Our study demonstrates that high-quality linkage of records belonging to different individuals in large routine databases from a middle-income country can be achieved, without unique identifiers. Importantly, for the purposes of establishing exposure to dengue for a casecontrol study, we were able to accurately identify links with a low false-match rate by using a bespoke linkage algorithm designed to overcome the challenges of linking different individuals in national data from Brazil. Although the restricted number of common variables for mothers and stillbirths limited the number of links that could be automatically detected, manual inspection allowed us to greatly improve sensitivity; however, this approach is resource-intensive and may not be feasible for larger databases, such as live births in Brazil with 3 million records a year. Our comparison of missed-matches and true-matches indicate that linkage errors occur randomly, and are unlikely to introduce bias into our analyses.

We show that linkage between large administrative datasets is complex and requires a number of steps. Our description of these steps (and commands listed in the annex) are available as guidance to others aiming to link similar data sources. Although we did not have a readily available training dataset (where the true match status of each record pair was known), we were able to create a gold-standard dataset from which to derive an appropriate match weight algorithm, and to evaluate the accuracy of both linkage methodologies. This was possible in our study because we had access to identifiable data, and could examine records manually, but is not always the case, when data from clinical and identifiers information are separated to protect the patient privacy [19].

The bespoke algorithm created specifically to link this dataset achieved higher linkage quality that the off-theshelf program RecLinkIII, which has been widely used to perform linkage in Brazil and which has previously been shown to have high sensitivity and specificity [7, 20-22]. RecLinkIII appears to work less well when there are a limited number of variables available to perform the linkage, as demonstrated in our study and a similar study by Coutinho et al. [23], which obtained a sensitivity of $60.9 \%$ and $72.8 \%$ (without and including the uncertain area respectively). The RecLinkIII program uses the Levenshtein string comparator, which according with Freire [24] is not the most effective option to compare names in Brazil. The Jaro-Winkler string comparator, used within our bespoke algorithm, has been shown to give the best results when compared with other string comparators to link names in Brazil [24]. Other string 
Table 4 Associations between linkage accuracy (using the bespoke algorithm) and characteristics of the cohort

\begin{tabular}{|c|c|c|c|c|c|c|c|}
\hline & $\begin{array}{l}\text { True matches } \\
N=123 \\
\mathrm{n}(\%)\end{array}$ & $\begin{array}{l}\text { Missed-matches } \\
N=68 \\
\mathrm{n}(\%)\end{array}$ & OR $(95 \% \mathrm{Cl})$ & $p$-value & $\begin{array}{l}\text { False-matches } \\
N=8 \\
\mathrm{n}(\%)\end{array}$ & OR $(95 \% \mathrm{Cl})$ & $p$-value \\
\hline \multicolumn{8}{|c|}{ Age of the mother in years } \\
\hline$<20$ & $25(20.3)$ & $19(27.9)$ & 1 & $p=0.047$ & - & & $p=0.095$ \\
\hline $20-35$ & $67(54.5)$ & $30(44.1)$ & $1.7(0.8-3.5)$ & & $8(100)$ & & \\
\hline$>35$ & $22(17.9)$ & $7(10.3)$ & $2.3(0.8-6.7)$ & & - & & \\
\hline Missing & $9(7.3)$ & $12(17.5)$ & & & - & & \\
\hline \multicolumn{8}{|l|}{ Maternal literacy } \\
\hline Illiterate & $7(5.7)$ & $5(7.3)$ & 1 & $p=0.113$ & - & 1 & $p=0.617$ \\
\hline $1-3$ years & $8(6.5)$ & $4(5.9)$ & $1.4(0.3-7.5)$ & & $1(12.5)$ & $0.9(0.4-1.9)^{a}$ & \\
\hline $4-7$ years & $32(26.0)$ & $14(20.6)$ & $1.6(0.4-6.0)$ & & $2(25.0)$ & & \\
\hline$>8$ years & $34(27.6)$ & $20(29.4)$ & $1.2(0.3-4.3)$ & & $4(50.0)$ & & \\
\hline$>11$ years & $19(15.4)$ & $3(4.4)$ & $4.5(0.8-24.1)$ & & - & & \\
\hline Missing & $23(18.7)$ & $22(32.3)$ & & & $1(12.5)$ & & \\
\hline \multicolumn{8}{|c|}{ Previous fetal death or abortion } \\
\hline No & $36(29.3)$ & $17(25.0)$ & 1 & $p=0.574$ & $2(25.0)$ & 1 & $p=0.966$ \\
\hline Yes & $59(48.0)$ & $31(45.6)$ & $0.9(0.4-1.8)$ & & $4(50.0)$ & $1.2(0.2-7)^{\mathrm{a}}$ & \\
\hline Missing & $28(22.7)$ & $20(29.4)$ & & & $2(25.0)$ & & \\
\hline \multicolumn{8}{|l|}{ Gestational age } \\
\hline$<22$ weeks & $9(7.3)$ & $2(2.9)$ & 1 & $p=0.248$ & - & & $p=0.492$ \\
\hline 22-27 weeks & 29 (23.6) & $18(26.5)$ & $0.3(0.1-1.8)$ & & $3(37.5)$ & 1 & \\
\hline 28-31 weeks & $21(17.8)$ & $11(16.2)$ & $0.4(0.1-2.3)$ & & $3(37.5)$ & $0.7(0.4-1.3)^{\mathrm{a}}$ & \\
\hline 32-36 weeks & $26(21.1)$ & $19(27.9)$ & $0.3(0.1-1.5)$ & & $2(25.0)$ & & \\
\hline 37-41 weeks & $28(22.8)$ & $9(13.2)$ & $0.7(0.1-3.8)$ & & - & & \\
\hline$\geq 42$ weeks & $2(1.6)$ & - & & & - & & \\
\hline Missing & $8(6.5)$ & $9(13.2)$ & & & - & & \\
\hline \multicolumn{8}{|c|}{ Birth or death weight } \\
\hline$\geq 2500$ & $23(18.7)$ & $16(23.5)$ & 1 & $p=0.798$ & - & & $p=0.165$ \\
\hline $1500-2500$ & $26(21.2)$ & $16(23.5)$ & $1.1(0.4-2.7)$ & & - & & \\
\hline$<1500$ & $64(52.0)$ & $31(45.6)$ & $1.4(0.6-3.0)$ & & $7(87.5)$ & & \\
\hline Missing & $10(8.1)$ & $7(7.3)$ & & & $1(12.5)$ & & \\
\hline
\end{tabular}

${ }^{a}$ Due to the small number of observations, we used only two categories, the first category without missing value as the reference one

comparators may be more appropriate in other situations [25]. The improved performance of the bespoke linkage algorithm was also likely due to our derivation of frequency-based match weights and the fact that we allowed weights to differ for common names and differences in age. Additional strengths of our approach were that the data were rigorously prepared to ensure that information belonging to the same mothers and babies could be linked; we performed the validation study in a large sample size that allowed evaluation of two thresholds and comparison with a commercially available software, and because we had additional information about the characteristics of the mothers included in the study we were able to check for bias.
An important aspect of linkage quality is the choice of threshold. The investigator, keeping in mind that different thresholds are more appropriate for different study questions, must make this choice. In our study, where linkage aimed to provide information on exposure (dengue during pregnancy) [26] we chose a conservative threshold to minimise false-matches. We showed that more relaxed thresholds added a number of false-matches, drastically decreasing the positive predictive value, without substantially increasing sensitivity. This is because stillbirth is a rare outcome, and there was a large number of real non-matches.

This study has a number of limitations. Although a gold-standard dataset was used to measure the linkage 
accuracy, there remains scope for linkage errors to occur: we may not have identified all missed-matches due to missing data on some records. Our estimates of sensitivity should therefore be interpreted with caution, and should not be assumed to apply to other datasets. Due to the low sensitivity of our linkage approach, our study should not be used to estimate stillbirth rates for women exposed to dengue during pregnancy. Our analysis of the association between study characteristics and linkage error may have been limited due to low power. A further consideration for case-control studies is whether linkage of cases and controls (to the exposure) has similar accuracy. We did not address the accuracy of linkage to live births in this study, but will evaluate this in future research.

\section{Conclusion}

To make best use of linked data, it is important to evaluate the quality of linkage processes and to understand the limitations and bias that errors in linkage could introduce in the research results [27, 28]. Validation studies are therefore useful for assessing whether probabilistic matching of such records is effective and whether results are reliable. We present this validation study to add to the limited existing evaluations of data linkage from middle income countries using a limited number of identifiers. We show that it is possible to achieve a high standard of linkage between different individuals within administrative data from Brazil, specifically for the purposes of accurately stillbirths exposed to dengue [26]. Our results highlight that bespoke linkage algorithms perform better than off-the-shelf software, and that manual review can be a valuable tool for improving sensitivity.

\section{Abbreviations}

PPV: Positive predictive value; Reclinkll: Software to link data used in Brazil; SIM: Mortality information system; SINAN: Notifiable diseases information system

\section{Acknowledgements}

The authors would like to thank Daniela Martins Neto, Tiago Mendes Cruz and Yara Rodrigues Flower for the valuable help in the data cleaning process.

\section{Funding}

EP funded by National Council for Scientific and Technological Development (CNPq-Brazil); LCR is partially funded by the European Union's Horizon 2020 research and innovation program under Zika- PLAN grant agreement No. 734584; KH is funded by the Wellcome Trust (grant number 103975/Z/14/Z) However the funder of this study had no role in study design, data collection, data analysis, data interpretation, or writing of the report.

\section{Availability of data and materials}

The data that support the findings of this study are available from Brazilian Ministry of Health but restrictions apply to the availability of these data, which were used under license for the current study, and so are not publicly available. Data are however available from the Brazilian Ministry of Health upon reasonable request.

\section{Authors' contributions}

EP carried out the analysis and wrote the first draft of the article. LR, MGT conceived the study. $\mathrm{KH}, \mathrm{KB}, \mathrm{MC}$ and RF contributed to the study design and interpretation. All authors revised the manuscript and approved the final version.

\section{Ethics approval and consent to participate}

Ethical approval was obtained from the Research Ethics Committee, Public Health Institute, Federal University of Bahia, Salvador, Brazil (CAAE: 26,797,814.7.0000.5030 CEP-ISC) and from London School of Hygiene and Tropical Medicine (Ethics Ref: 10,269).

\section{Consent for publication}

Not applicable.

\section{Competing interests}

The authors declare that they have no competing interests.

\section{Publisher's Note}

Springer Nature remains neutral with regard to jurisdictional claims in published maps and institutional affiliations.

\section{Author details}

'London School of Hygiene and Tropical Medicine, Keppel St, Bloomsbury, London WC1E 7HT, UK. ${ }^{2}$ Instituto de Saúde Coletiva, Rua Basílio da Gama, s/ n.Canela, Salvador, Bahia CEP 40110040, Brazil. ${ }^{3}$ Departamento de Estatística, Av Ademar de Barros, s/n Ondina, Salvador, Bahia CEP 40170110, Brazil.

Received: 10 April 2017 Accepted: 10 July 2017

Published online: 17 July 2017

\section{References}

1. Sayers A, Ben-Shlomo Y, Blom AW, Steele F. Probabilistic record linkage. Int J Epidemiol. 2016;45(3):954-64. doi:10.1093/ije/dyv322.

2. Jutte DP, Roos LL, Brownell MD. Administrative record linkage as a tool for public health research. Annu Rev Public Health. 2011;32:91-108.

3. Harron K, Gilbert R, Cromwell D, van der Meulen J. Linking data for mothers and babies in de-identified electronic health data. PLoS One. 2016;11(10): e0164667.

4. Ford JB, Roberts CL, Taylor LK. Characteristics of unmatched maternal and baby records in linked birth records and hospital discharge data. Paediatr Perinat Epidemiol. 2006;20:329-37.

5. Liu C, Cnattingius S, Bergström M, Östberg V, Hjern A. Prenatal parental depression and preterm birth: a national cohort study. BJOG. 2016; n/a-n/a, doi: 10.1111/1471-0528.13891.

6. Kamphuis $\mathrm{E}$, et al. Fetal gender of the first born and the recurrent risk of spontaneous preterm birth. Am J Obstet Gynecol. 2015;212:S386.

7. Fonseca MGP, Coeli CM, Lucena F. De F de a, Veloso VG, Carvalho MS. accuracy of a probabilistic record linkage strategy applied to identify deaths among cases reported to the Brazilian AIDS surveillance database. Cad Saúde Pública. 2010;26(7):1431-8.

8. Kariminia A, Butler T, Corben S, Kaldor J, Levy M, Law M. Mortality among prisoners: how accurate is the Australian National Death Index? Aust N Z Public Health. 2005;29(6):572-5.

9. Clark DE. Practical introduction to record linkage for injury research. Inj Prev. 2004:10(3):186-91.

10. Newcombe HB, Kennedy JM, Axford SJ, James AP. Automatic linkage of vital records. In: Record linkage techniques; 1985.

11. Harron K. Evaluating data linkage techniques for the analysis of bloodstream infection in paediatric intensive care (PhD Thesis). University College London; 2014

12. Harron K, Goldstein H, Wade A, Muller-Pebody B, Parslow R, Gilbert R. Linkage, evaluation and analysis of national electronic healthcare data: application to providing enhanced blood-stream infection surveillance in paediatric intensive care. 2013 [cited 13 Oct 2015]; Available from: http://dx. plos.org/10.1371/journal.pone.0085278.

13. Moore CL, Amin J, Gidding HF, Law MG. A new method for assessing how sensitivity and specificity of linkage studies affects estimation. PLoS One. 2014;9:e103690.

14. Brazil. Ministry of Health of Brazil. Nova classificacão de dengue. [cited 13 Oct 2015]; Available from: http://www.epi.uff.br/wp-content/uploads/2013/ 10/Nova_classificacao_de_caso_de_dengue_OMS.pdf 
15. Brazil. Ministry of Health of Brazil. Manual de vigilância do óbito infantil e fetal e do comitê de prevenção do óbito infantil e fetal. MS Braślia; 2009. [cited 13 Oct 2016]; available from: http://bvsms.saude.gov.br/bvs/ publicacoes/manual_obito_infantil_fetal_2ed.pdf.

16. Yancey WE. Evaluating string comparator performance for record linkage. Statiscal Research Division; U. S. Census Bureau; Washington, DC.

[cited 13 Oct 2016]; Available from: https://www.census.gov/srd/papers/ $\mathrm{pdf} / \mathrm{rrs} 2005-05 . \mathrm{pdf}$.

17. Levenshtein VI. Binary codes capable of correcting deletions, insertions and reversals. Soviet Physics Dokl. 1966;10:707-10.

18. de Camargo KR Jr, Coeli CM. Reclink: aplicativo para o relacionamento de bases de dados, implementando o método probabilistic record linkage. Cad Saúde Pública. 2000;16(2):439-47.

19. Kelman CW, Bass AJ, Holman CDJ. Research use of linked health data-a best practice protocol. Aust N Z J Public Health. 2002:26(3):251-5.

20. TLN d S, Klein CH, da Rocha Nogueira A, LHA S, NAS e S, Bloch KV. Cardiovascular mortality among a cohort of hypertensive and normotensives in Rio de Janeiro-Brazil-1991-2009. BMC Public Health. 2015;15(1):1.

21. Coutinho ESF, Coeli CM. Accuracy of the probabilistic record linkage methodology to ascertain deaths in survival studies. Cad Saúde Pública. 2006;22(10):2249-52.

22. De Oliveira GP, Bierrenbach AL de S, de Camargo KR, Coeli CM, Pinheiro RS. Accuracy of probabilistic and deterministic record linkage: the case of tuberculosis. Revista de Saúde Pública. 2016;50:49. doi:10.1590/S1518-8787. 2016050006327

23. Coutinho RG, da M, Coeli CM, Faerstein E, Chor D. Sensitivity of probabilistic record linkage for reported birth identification: Pró-Saúde study. Rev Saude Publica. 2008:42(6):1097-100.

24. Freire SM, Gonçalves R de CB, Bandarra AC, Villela MGT, Meire A, Cabral $M D B$, et al. Análise da efetividade de comparadores de strings para discriminar pares verdadeiros de pares falsos no relacionamento de registro. In: Anais do IX Workshop de Informática Médica XXIX Congresso da Sociedade Brasileira de Computação-IX Workshop de Informática Médica Bento Gonçalves: Sociedade Brasileira de Computação [Internet]. 2009 [cited 24 Nov 2016]. p. 2119-2128.

25. Grannis S, Overhage J, McDonald C. Real world performance of approximate string comparators for use in patient matching. Stud Health Technol Inform. 2004;107:43-7.

26. Paixao Es, Costa MCN, Teixeira MG, Harron K, Almeida MF, Barreto ML, Rodrigues LC. Symptomatic dengue during pregnancy and the risk of stillbirth: a matched case control study using routine data in Brazil (20062012). Lancet Infect Dis. 2017. (in press).

27. Harron K, Wade A, Gilbert R, Muller-Pebody B, Goldstein H. Evaluating bias due to data linkage error in electronic healthcare records. BMC Med Res Methodol. 2014;14:36.

28. Bohensky $\mathrm{M}$, et al. Data linkage: a powerful research tool with potential problems. BMC Health Serv Res. 2010;10:346-52.

\section{Submit your next manuscript to BioMed Central and we will help you at every step:}

- We accept pre-submission inquiries

- Our selector tool helps you to find the most relevant journal

- We provide round the clock customer support

- Convenient online submission

- Thorough peer review

- Inclusion in PubMed and all major indexing services

- Maximum visibility for your research

Submit your manuscript at www.biomedcentral.com/submit

CBiomed Central 